\title{
Elastic heterogeneity, vibrational states, and thermal conductivity across an amorphisation transition
}

\author{
Hideyuki Mizuno $^{1}$ (a), Stefano Mossa ${ }^{2}$ (b) and Jean-Louis Barrat ${ }^{1}$ (c) \\ 1 Laboratory for Interdisciplinary Physics, UMR 5588, Université Grenoble 1 and CNRS, 38402 Saint Martin d'Hères, \\ France \\ 2 INAC/SPrAM (UMR 5819 UJF, CNRS, CEA), CEA-Grenoble, 17 Rue des Martyrs, 38054 Grenoble, France
}

PACS 62.25.-g-Mechanical properties of nanoscale systems

PACS 63.50. $-\mathrm{x}$ - Vibrational states in disordered systems

PACS 65.60.+a - Thermal properties of amorphous solids and glasses: heat capacity, thermal expansion, etc.

\begin{abstract}
Disordered solids exhibit unusual properties of their vibrational states and thermal conductivities. Recent progresses have well established the concept of "elastic heterogeneity", i.e., disordered materials show spatially inhomogeneous elastic moduli. In this study, by using molecular-dynamics simulations, we gradually introduce "disorder" into a numerical system to control its modulus heterogeneity. The system starts from a perfect crystalline state, progressively transforms into an increasingly disordered crystalline state, and finally undergoes structural amorphisation. We monitor independently the elastic heterogeneity, the vibrational states, and the thermal conductivity across this transition, and show that the heterogeneity in elastic moduli is well correlated to vibrational and thermal anomalies of the disordered system.
\end{abstract}

Introduction. - Disordered solids, including structural glasses, disordered crystals, or even crystals with complex unit cells, have unusual vibrational and thermal properties [1,2] compared to those of the corresponding crystalline materials, which are well understood by the Debye model. The density of states $g(\omega)$ shows an excess over the Debye prediction $g(\omega) \sim \omega^{2}$, often referred to as the "Boson peak" in glasses, in a frequency regime of $\omega \sim 1[T H z]$. The excess in $g(\omega)$ is mirrored by an excess in the specific heat $C(T)$ over the prediction $C \sim T^{3}$. The $T$-dependence of the thermal conductivity $\kappa(T)$ is also very different from that of crystals, with a characteristic "plateau" around $T \sim 10[K]$.

The origin of the unusual features has been interpreted within several different approaches: soft anharmonic nature of the potentials 3, evolution from a van Hove singularity [4, crossover from a minima-dominated phase to a saddle-point-dominated phase [5], proximity of a "jamming" singularity [6, or existence of elastic heterogeneities 7 7 9]. The concept of of elastic heterogeneity in amorphous materials has been well established thanks

\footnotetext{
(a) E-mail: Hideyuki.Mizuno@ujf-grenoble.fr

(b) E-mail: stefano.mossa@cea.fr

(c) E-mail: jean-louis.barrat@ujf-grenoble.fr
}

to a series of simulation studies $10-12$. Contrary to crystals, amorphous materials exhibit spatially inhomogeneous elastic moduli, with coexistence of hard and soft regions on the scale of a few tenths of particles. Soft regions exhibit even negative elastic moduli 10 , which are stabilized by surrounding stable regions with positive moduli. It is natural that such modulus heterogeneities induce anomalies in vibrational states and thermal properties, and several works have attempted to clarify this connection. Leonforte et al. [13] studied the spatial structure of non-affine displacement fields under deformation, which reflects the elastic heterogeneity, and found a correlation between that structure and the Boson peak frequency. Schirmacher et al. 8, 9 proposed a theoretical model, where random spatial fluctuation of shear modulus determines the Boson peak and even the anomalous temperature dependence of thermal conductivity. A more recent study 14 showed qualitative agreement between this model and simulation results.

The aim of this work is to investigate in a more systematic manner this connection, by studying a system in which the heterogeneous nature of the elastic response can be varied continuously by tuning a control parameter. This is achieved here by progressively introducing a size 
disorder in a crystalline system, in such a way that the system eventually undergoes structural amorphisation, as shown in Ref. [15]. The elastic heterogeneity, vibrational states, and thermal conductivity are monitored simultaneously across this evolution towards the amorphous state.

System description. - We start from an onecomponent, perfect face-centered cubic (FCC) crystal in 3 -dimensions. Particles $i$ and $j$ interact via a soft-core potential $\phi^{i j}=\epsilon\left(\sigma^{i j} / r^{i j}\right)^{12}$ with $\sigma^{i j}=\left(\sigma^{i}+\sigma^{j}\right) / 2$, where $\sigma^{i}$ and $\sigma^{j}$ are their diameters, and $r^{i j}$ is the distance between them. The potential is cut off and shifted to zero at $r^{i j}=2.5 \sigma^{i j}$. In the initially monodisperse system, $\sigma^{i}=\sigma$ for all particles. Owing to the scaling property of the inverse power potential [16], the thermodynamic state depends on a single parameter $\Gamma=\hat{\rho}\left(\epsilon / k_{B} T\right)^{1 / 4} \sigma^{3}$, where $k_{B}$ is Boltzmann constant, $T$ is the temperature, and $\hat{\rho}=N / V$ is the number density. In the following, $\sigma, \epsilon / k_{B}$, and $\tau=\left(m \sigma^{2} / \epsilon\right)^{1 / 2}$ (where $m$ is the particle mass) are used as units of length, temperature, and time, respectively. We have set $\hat{\rho}=1.015$ and the length of the unit cell of the FCC crystal is $a=1.58$. Most part of our simulations used a system of $N=4,000$ particles, of linear dimension $L=10 a=15.8$. For the calculation of the vibrational states, larger systems with $L$ ranging from $L=12 a(N=6,912)$ to $30 a(N=108,000)$ were also considered. The FCC crystalline system was equilibrated at $T=0.01(\Gamma=3.21)$ by using a $N V T$ MD simulation with periodic boundary conditions. For the monodisperse system, the melting and glass transition temperatures have been reported to be $T_{m} \simeq 0.6\left(\Gamma_{m} \simeq 1.15\right)$ and $T_{g} \simeq 0.2$ $\left(\Gamma_{g} \simeq 1.5\right)$, respectively 16 .

Disorder was introduced into the crystalline state as described in Ref. 15. Half of the particles, randomly selected, are assigned to species 1 , the remaining half constitute component 2 . The size $\sigma_{1}$ of species 1 is gradually reduced below its initial value $1[\sigma]$, while at the same time, the size $\sigma_{2}$ of 2 is increased. During the process, an "effective diameter" $\sigma_{\text {eff }}$ of the binary mixture is held fixed at the initial value $1[\sigma]$. In an approximate one-component description, $\sigma_{\text {eff }}$ is given by $\sigma_{\text {eff }}^{3}=\sum_{\alpha, \beta=1,2} x_{\alpha} x_{\beta} \sigma_{\alpha \beta}^{3}[16$, where $x_{1}=x_{2}=1 / 2$ are the fractions of the two components. $\lambda=\sigma_{1} / \sigma_{2} \leq 1$ measures the degree of disorder, and uniquely determines $\sigma_{1}$ and $\sigma_{2}$ using the condition $\sigma_{\text {eff }}^{3} \equiv 1$. The coupling parameter $\Gamma$ is replaced by $\Gamma=\hat{\rho}\left(\epsilon / k_{B} T\right)^{1 / 4} \sigma_{\text {eff }}^{3}$ for the binary mixture, and held fixed at the initial large value $\Gamma \equiv 3.21$. In the following calculations, $\lambda$ was decreased starting from the ideal crystal $(\lambda=1)$ by a succession of small steps, $\Delta \lambda=10^{-4}$. After each change $\Delta \lambda$, the system was re-equilibrated using a NVT MD simulation.

Upon decreasing $\lambda$, the system undergoes a discontinuous transition toward an amorphous state, as observed (in 2-dimensions) in Ref. 15] The transition point $\lambda^{*}$ is located by monitoring two order parame-

\footnotetext{
${ }^{1}$ Note that this cannot be considered as a genuine first order phase transition. See Ref. [15] for details.
}
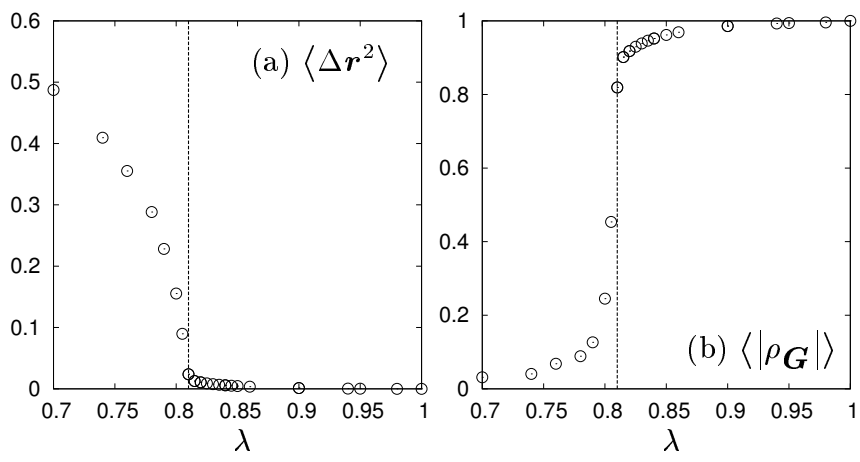

Fig. 1: Order parameters across the amorphisation transition. (a) $\left\langle\Delta \boldsymbol{r}^{2}\right\rangle$ and (b) $\left\langle\left|\rho_{\boldsymbol{G}}\right|\right\rangle$, versus $\lambda$. The system undergoes a transition from crystal to amorphous state at $\lambda=\lambda^{*} \simeq 0.81$, indicated by vertical lines.

ters, $\left\langle\Delta r^{2}\right\rangle=(1 / N) \sum_{j=1}^{N}\left(\left\langle\boldsymbol{r}^{j}\right\rangle-\boldsymbol{r}_{0}^{j}\right)^{2}$ and $\left\langle\left|\rho_{\boldsymbol{G}}\right|\right\rangle=$ $\left\langle\left|(1 / N) \sum_{j=1}^{N} \exp \left(i \boldsymbol{G} \cdot \boldsymbol{r}^{j}\right)\right|\right\rangle$. Here, \langle\rangle denotes a time average, $\boldsymbol{r}^{j}$ is the instantaneous position of $j$ th particle, $\boldsymbol{r}_{0}^{j}$ is the FCC lattice site, and $\boldsymbol{G}$ is the smallest reciprocal lattice vector, $\boldsymbol{G}=(2 \pi / a, 2 \pi / a,-2 \pi / a)$. Fig. 1 shows $\left\langle\Delta r^{2}\right\rangle$ and $\left\langle\left|\rho_{\boldsymbol{G}}\right|\right\rangle$ versus $\lambda$. The transition takes place at $\lambda \simeq 0.81$, where both $\left\langle\Delta r^{2}\right\rangle$ and $\left\langle\left|\rho_{\boldsymbol{G}}\right|\right\rangle$ exhibit discontinuous jumps. We confirmed that $\lambda^{*}$ does not depend on the initial repartition of the two species on the lattice. For $1>\lambda \geq \lambda^{*} \simeq 0.81$, the system is in a chemically disordered crystalline state, with non-zero Bragg peaks for the number density. For $\lambda^{*}>\lambda \geq 0.7$, the system is in an amorphous state, with complete loss of periodicity.

Elastic heterogeneity. - Inhomogeneous systems like glasses or complex crystals exhibit heterogeneous, scale-dependent distributions of local elastic moduli, which can be determined using several methods [10 12 17]. Here, the system was divided into $20 \times 20 \times 20$ cubic domains with a linear size $W=2 a=3.16$, and the local elastic moduli were obtained from equilibrium fluctuation formulae 18. (Details about the calculations are included in Supplemental Material 19.) For each subvolume, one local bulk modulus and five local shear moduli are defined, corresponding to the response to isotropic bulk compression and volume-conserving shear deformations, respectively [17]. Of the five shear moduli, two correspond to "pure" shear deformations (plane and triaxial strain deformations), and the remaining three are related to "simple" shear deformations. In the following, the local bulk, pure shear, and simple shear moduli are denoted by $K^{m}$, $G_{p}^{m}$, and $G_{s}^{m}$, respectively (where "m" indicates a local quantity).

For each local modulus $C^{m}=K^{m}, G_{p}^{m}, G_{s}^{m}$, the probability distribution function $P\left(C^{m}\right)$, which turns out to be closely Gaussian, was obtained by sampling the different cubic subvolumes. Note that $P\left(G_{p}^{m}\right)$ and $P\left(G_{s}^{m}\right)$ are different in cubic crystals, while they coincide with each other in isotropic glasses. Each modulus is the sum of four contributions, the Born term $C_{B}^{m}$, the kinetic con- 

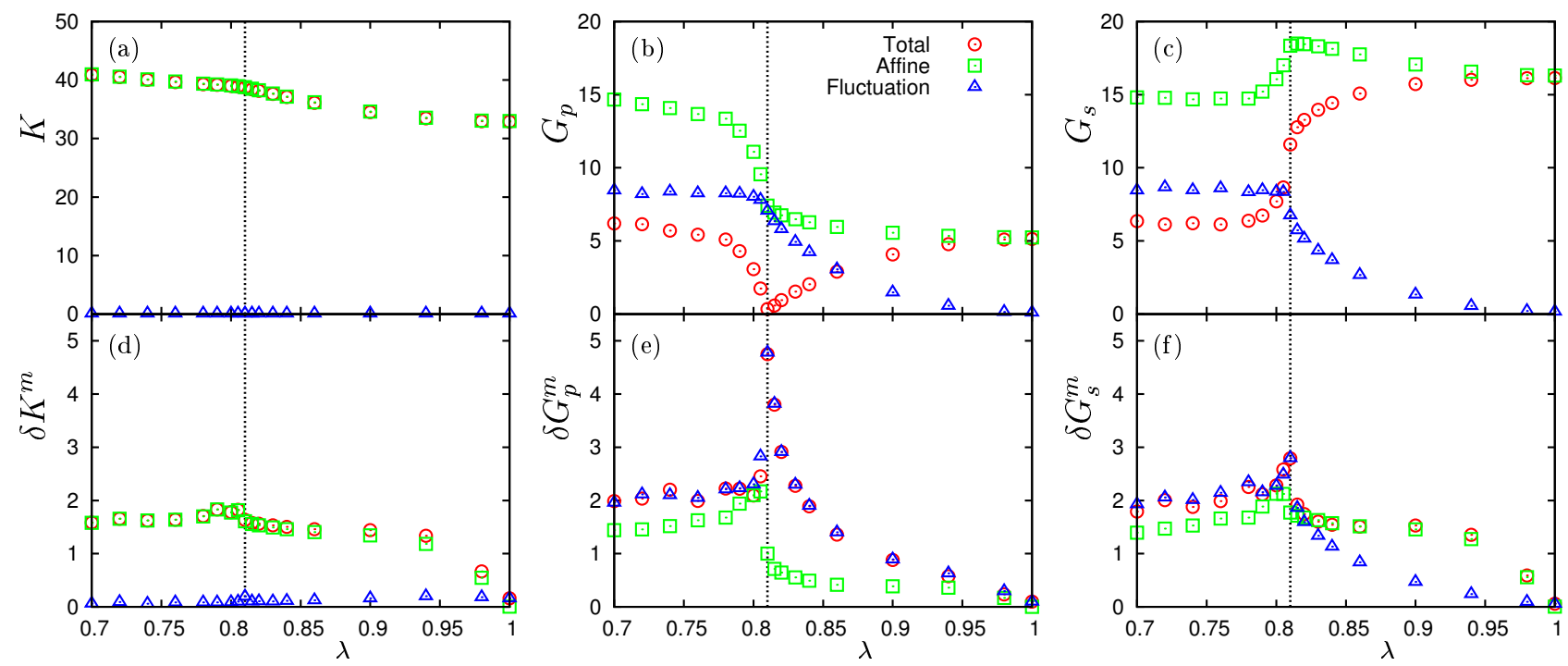

Fig. 2: The macroscopic values (average values), (a) $K$, (b) $G_{p}$, and (c) $G_{s}$, and the standard deviations, (d) $\delta K^{m},(\mathrm{e}) \delta G_{p}^{m}$, and (f) $\delta G_{s}^{m}$, versus the disorder parameter $\lambda$. Circles, squares, and triangles represent values of the total modulus $C^{m}$, the affine term $C_{A}^{m}$, and the fluctuation term $C_{F}^{m}$, respectively. The vertical lines indicate the transition point $\lambda=\lambda^{*} \simeq 0.81$.

tribution $C_{K}^{m}$, the pressure correction term $C_{C}^{m}$, and the fluctuation term $-C_{F}^{m}$ (See Supplemental Material [19]). The sum of the first three terms, denoted by $C_{A}^{m}$ in Fig. 2. corresponds to the response of a system which deforms affinely at all scales; The fluctuation term $-C_{F}^{m}$ is a negative correction that accounts for the non-affinity of the deformation at small scales, and in disordered systems becomes comparable in magnitude to $C_{A}^{m}$ 20. The average value, $C=\int C^{m} P\left(C^{m}\right) d C^{m}$, and standard deviation, $\delta C^{m}=\sqrt{\int\left(C^{m}-C\right)^{2} P\left(C^{m}\right) d C^{m}}$, are reported in figure 2. The average value, $C=K, G_{p}, G_{s}$, coincides with the "macroscopic" modulus, denoted without "m". In the initial crystalline state $\lambda=1, K>G_{s}>G_{p}$, while $K>G_{p} \simeq G_{s}$ for the isotropic amorphous states $\lambda \leq 0.78$. For a system with inverse power interactions, the bulk modulus $K$ is determined by the affine $K_{A}$ only, i.e., $K=K_{A}\left(K_{F}=0\right)$ (see Fig. 2(a)). On the other hand, the non-affine components, $G_{p F}$ and $G_{s F}$, get progressively larger with decreasing $\lambda$. Remarkably, around the transition $\lambda^{*}, G_{p F}$ reaches the affine $G_{p A}$, so that the total $G_{p}$ vanishes, $G_{p} \simeq 0$. Therefore, the transition can be described as an elastic instability associated with the shear modulus $G_{p}$. Below the transition $\left(\lambda<\lambda^{*}\right)$, the material becomes rapidly isotropic, as it is manifested by the convergence $G_{p} \simeq G_{s}$.

Across the amorphisation transition, the elastic heterogeneity, characterized by the standard deviation of the moduli, undergoes important changes, starting from a spatially homogeneous modulus distribution at $\lambda=1$. As $\lambda$ decreases from 1 to $0.9, \delta K^{m}$ and $\delta G_{s}^{m}$ increase monotonically, mainly in relation to an increase in $\delta K_{A}^{m}$ and $\delta G_{s A}^{m}$, respectively. In the same range, $\delta G_{p}^{m}$ is already dominated by the heterogeneity in the fluctuation term $\delta G_{p F}^{m}$. As $\lambda$ approaches $\lambda^{*}$, this heterogeneity increases rapidly, and
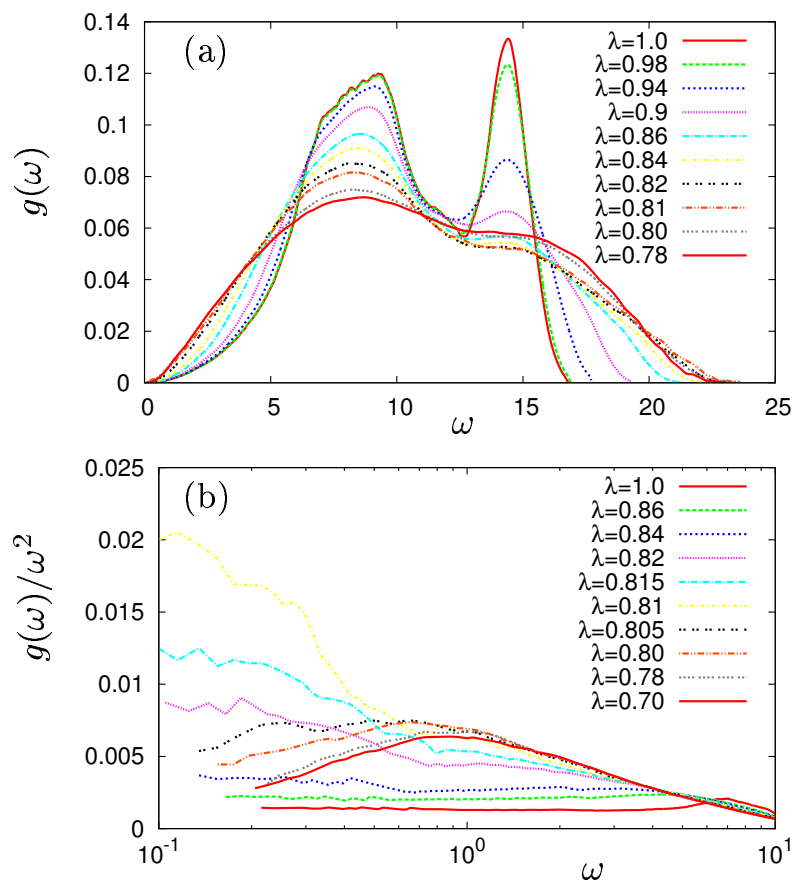

Fig. 3: (a) Density of states $g(\omega)$ and (b) reduced density of states $g(\omega) / \omega^{2}$ for various $\lambda$ s.

the spatial distribution of $G_{p}^{m}$ becomes extremely heterogeneous with a nearly zero average value, $G_{p} \simeq 0$ and a large standard deviation, $\delta G_{p}^{m} \simeq 5$. Below the transition point $\lambda^{*}, \delta G_{p}^{m}$ and $\delta G_{s}^{m}$ immediately converge to a very similar value, $\delta G_{p, s}^{m} \simeq 2$. In the following, these changes in elastic properties will be correlated with the evolution of the vibrational and thermal properties.

Vibrational states. - A standard normal modes analysis was performed by diagonalizing the Hessian ma- 


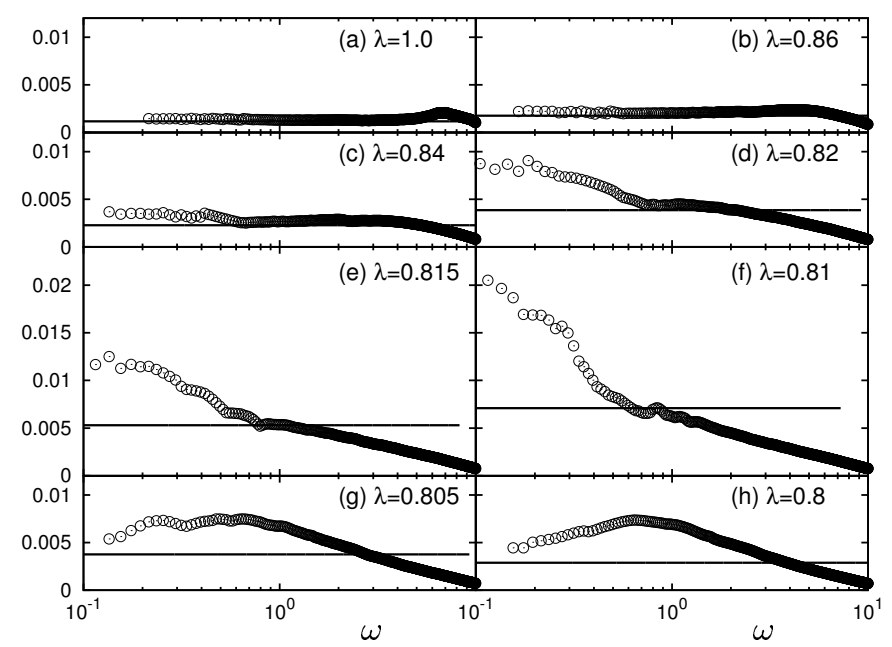

Fig. 4: Reduced density of state $g(\omega) / \omega^{2}$ compared to the Debye-model prediction $g_{D}(\omega) / \omega^{2}=3 / \omega_{D}^{3}$. Symbols and lines represent $g(\omega) / \omega^{2}$ and $g_{D}(\omega) / \omega^{2}$, respectively.

trix for all considered values of the disorder parameter, in the range $1 \geq \lambda \geq 0.7$. The resulting vibrational densities of states (VDOS) $g(\omega)$ are shown in figure 3(a). In the crystalline state $\lambda=1$, a "transverse branch" in the range $\omega \in[7: 9.5]$ and a "longitudinal branch" around $\omega=14.5$ can be clearly identified in the VDOS. As $\lambda$ decreases, these contributions from well identified phonon branches tend to loose their identity. In particular, the peak associated with the longitudinal branch is already suppressed at $\lambda=0.9$, which is correlated with the appearance of significant heterogeneities of the bulk modulus $K^{m}$ and of the shear modulus $G_{s}^{m}$. These heterogeneities induce a broadening of the high $\omega$ modes, which leads to the reduction of the intensity of the longitudinal peak.

The heterogeneity in low shear modulus $G_{p}^{m}$, on the other hand, is well correlated with the behaviour of the VDOS in the low $\omega$ region. This correlation is most easily seen by considering the reduced VDOS, $g(\omega) / \omega^{2}$, shown in Figs. 3(b) and 4. In Fig $4, g(\omega) / \omega^{2}$ is compared to the Debye-model prediction, $g_{D}(\omega) / \omega^{2}=3 / \omega_{D}^{3}$, where the Debye frequency $\omega_{D}$ is calculated from the macroscopic moduli, $K, G_{p}$, and $G_{s} 21$. At $1 \geq \lambda \geq 0.84, g(\omega) / \omega^{2}$ coincides with $g_{D}(\omega) / \omega^{2}$, indicating that low $\omega$ excitations are not affected by the modulus heterogeneities. An excess of modes in $g(\omega) / \omega^{2}$ over $g_{D}(\omega) / \omega^{2}$ starts to appear around $\lambda=0.82$. This excess is particularly pronounced at very low frequencies, and increases with the spatial heterogeneity of $G_{p}^{m}$ as $\lambda$ approaches $\lambda^{*}$. Below the transition $\lambda^{*}$, the excess of modes drops immediately, accompanying a rapid decrease in the heterogeneity of $G_{p}^{m}$. At $\lambda \leq 0.8$, a typical Boson peak, with an amplitude comparable to what is typically observed in soft sphere or Lennard-Jones 22 glasses, is formed around $\omega=\omega_{\mathrm{BP}} \simeq 1$. Note that the excess of modes in the disordered crystals close to $\lambda^{*}$ is observed at much lower frequencies, presumably down to zero frequency at the transition point,

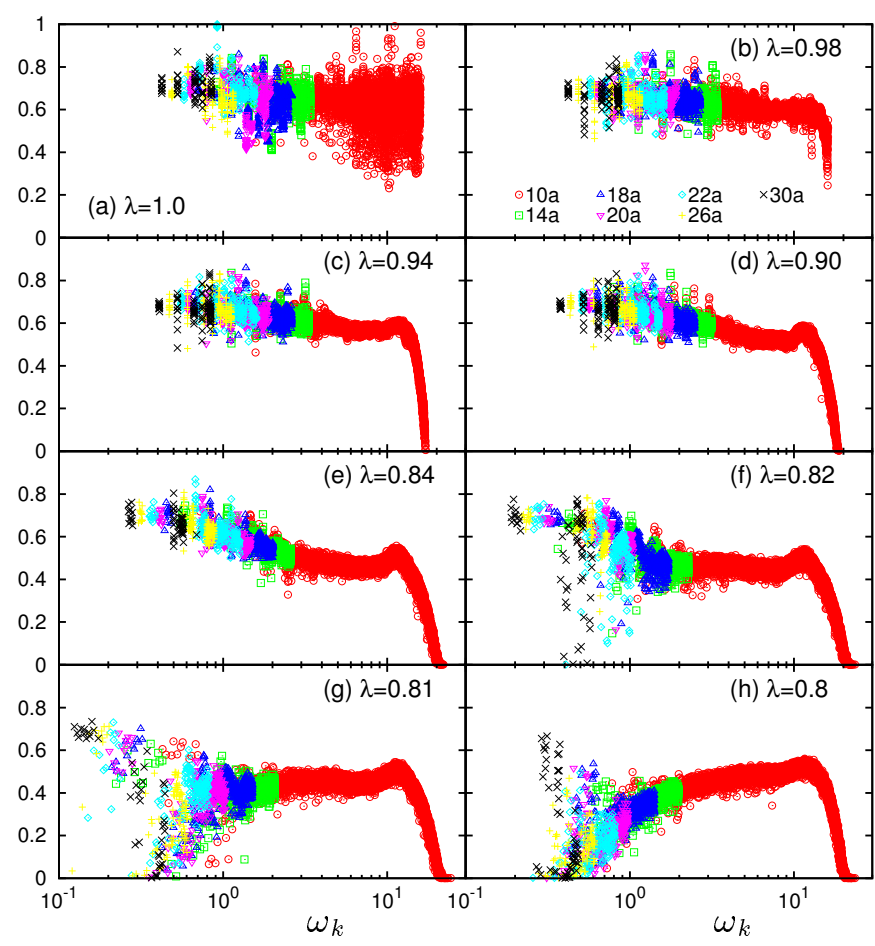

Fig. 5: Participation ratio $P R_{k}$ versus $\omega_{k}$. To access lower $\omega_{k}$ modes, several systems with sizes $L$ ranging from $L=10 a$ $(N=4,000)$ to $30 a(N=108,000)$ have been considered.

in relation to the vanishing of $G_{p}$ at the transition. Altogether, the position and amplitude of the peak appear to be controlled by the shear modulus average value and heterogeneity, in the disordered crystal as well as in the amorphous state. This is qualitatively consistent with the theoretical ideas of Refs. 8, 9, and a more quantitative analysis will be presented in future work.

The participation ratio, $P R_{k}=$ $(1 / N)\left[\sum_{j=1}^{N}\left(\boldsymbol{e}_{k}^{j} \cdot \boldsymbol{e}_{k}^{j}\right)^{2}\right]^{-1}$, where $k$ is the eigen-mode number, and $\boldsymbol{e}_{k}^{j}$ is the polarization vector, is shown with the eigen-frequency $\omega_{k}$ in Fig. 5. (See Supplemental Material 19 for the eigen-vectors spatial structure.) As extreme cases, $P R_{k}=2 / 3$ for an ideal standing plane wave and $P R_{k} \simeq 1 / N$ for an ideal mode involving only one particle 23]. As $\lambda$ decreases from 1 to 0.9 , high $\omega$ modes become localized with low $P R_{k}$ values. This corresponds to the disappearance of the longitudinal peak in the VDOS, and can be related to the heterogeneities of $K^{m}$ and $G_{s}^{m}$. On the other hand, low $\omega$ quasi-localized modes 24] start to become apparent at $\lambda \leq 0.82$. At $\lambda=0.8$, i.e., in the amorphous state, the result is consistent with that reported for a Lennard-Jones glass [23]. Clearly, the appearance of these quasi-localized modes is correlated with that of an excess VDOS, and to the sharp increase in the heterogeneity of $G_{p}^{m}$. This confirms the recent findings in simulation 25] and experiment 26 which demonstrated the transverse nature of low frequency excess modes, which were assigned to the coupling of transverse vibrational modes with defective 

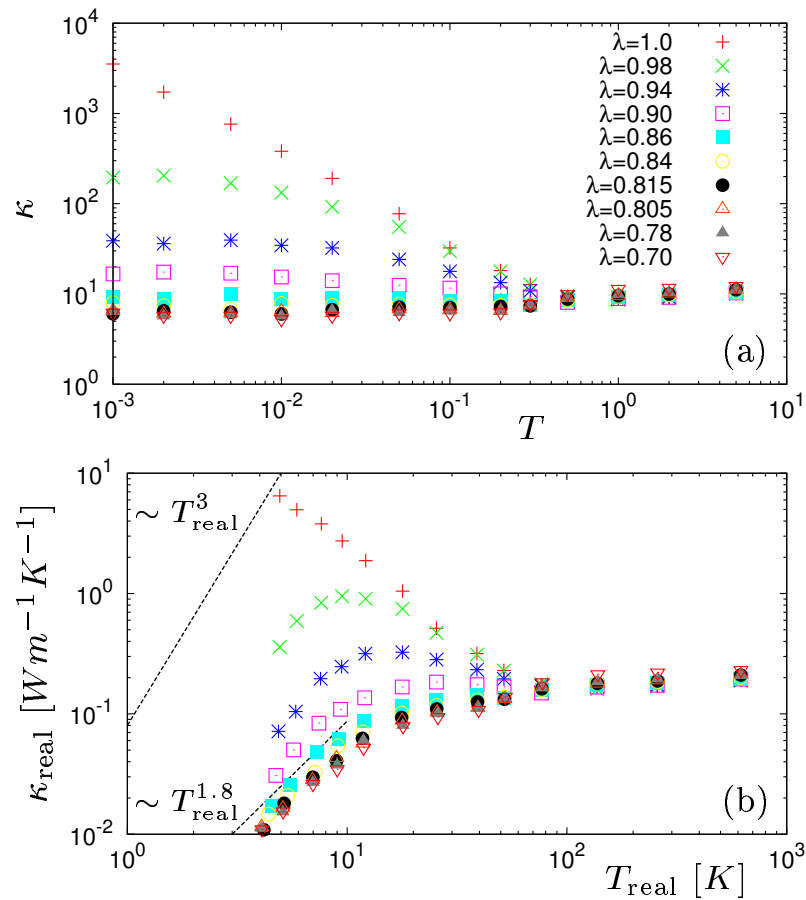

Fig. 6: The temperature dependence of thermal conductivity $\kappa$ for $\lambda=1$ to 0.7 . (a) The values obtained from MD simulations and (b) the values with quantum corrections. In (b), $T_{\text {real }}$ and $\kappa_{\text {real }}$ are measured in units of $K$ and $W m^{-1} K^{-1}$, respectively.

soft structures.

Thermal conductivity. - The thermal conductivity $\kappa$ was obtained from NVE MD simulations, using the Green-Kubo formulation 27: $\kappa=$ $\left(1 / 3 k_{B} T^{2} V\right) \int_{0}^{\infty}\langle\boldsymbol{J}(t) \cdot \boldsymbol{J}(0)\rangle d t$, where $\boldsymbol{J}$ is the heat current vector. Figure 6(a) shows the temperature dependence of $\kappa$ at the indicated values of $\lambda$. In the crystalline state $\lambda=1, \kappa$ decreases with increasing $T$, due to the increase of anharmonicity. As $\lambda$ decreases, the disorder scatters heat carriers and reduces $\kappa$ steeply. The reduction of $\kappa$ saturates around $\lambda=0.86$, i.e., before a significant excess of modes is observed in the VDOS. For $\lambda \leq 0.86, \kappa$ is insensitive to $T$, which means that disorder dominates anharmonic effects.

The above behavior of $\kappa$ can be rationalized by using the simple formula: $\kappa=(1 / 3) C v \ell$ [27, where $C$ is the heat capacity per unit volume, $v$ and $\ell$ are the "average" velocity and mean free path of the heat carriers, respectively. $C$ and $v$ are taken as constants, and the attenuation rate $\sim 1 / \ell$, a function of $T$ and $\lambda$, is decomposed into two components, anharmonic $1 / \ell_{\text {anh }}$ and disorder $1 / \ell_{\text {dis }}$, which respectively depend on $T$ and $\lambda$ : $1 / \ell(T, \lambda)=1 / \ell_{\operatorname{anh}}(T)+1 / \ell_{\operatorname{dis}}(\lambda) 28.1 / \ell_{\text {dis }}$ is expected to be increased by the modulus heterogeneities. In the perfect crystal $(\lambda=1), 1 / \ell_{\mathrm{anh}} \gg 1 / \ell_{\mathrm{dis}}$, leading to $\ell \simeq \ell_{\mathrm{anh}}$. In this case, the anharmonic effect reduces $\ell$ with increasing $T$ and causes the reduction of $\kappa$. As $\lambda$ decreases, $1 / \ell_{\text {dis }}$ becomes large, and $1 / \ell_{\text {anh }} \ll 1 / \ell_{\text {dis }}$ at $\lambda \leq 0.86$. Comparing with the results reported in Fig. 2 it appears that the increase of $1 / \ell_{\text {dis }}$ is mainly correlated to the heterogeneities of $K^{m}$ and $G_{s}^{m}$. At $\lambda=0.86$, we measured $\ell=3 \kappa / C v \sim 1[\sigma]$, the order of particle diameter, i.e., $\ell$ and $\kappa$ reach a "minimum" value, insensitive to temperature. At this stage, even if further disorder makes local moduli more heterogeneous, $\kappa$ does not decrease any more. Actually, in such a strong scattering state with an extremely short $\ell$, heat carriers are no longer propagating phonons, but non-propagating or quasi-stationary excitations, sometimes called diffusons [29].

In the low temperature regime, the calculations presented above are based on a classical approach, where the heat capacity $C$ is independent of $T$. However, in a real system, $C$ depends on $T$, e.g., $C \sim T^{3}$ for the Debye model, due to quantum effects. In order to assess qualitatively how the results would be modified by quantum effects, a simple quantum correction, often used in previous works [27, was applied. (See Supplemental Material 19 for details.) The correction maps "classical" values of $T_{\mathrm{MD}}$ and $\kappa_{\mathrm{MD}}$, obtained from MD simulations, onto "real" values of $T_{\text {real }}$ and $\kappa_{\text {real }}$. The mapping of $\kappa_{\mathrm{MD}}$ onto $\kappa_{\text {real }}$ is $\kappa_{\text {real }}=\left(C_{\text {real }} / C_{\mathrm{MD}}\right) \kappa_{\mathrm{MD}}$, which takes into account the "real" $T$ dependence of the heat capacity $C_{\text {real }}$. Note that $C_{\text {real }}$ can be calculated from the density of state $g(\omega)$. Temperature mapping can be performed in two ways, with or without inclusion of the "zero point energy". In the present study, the correction without inclusion of the zero point energy was applied [27.

Figure 6(b) shows the $T_{\text {real }}$ dependence of $\kappa_{\text {real }}$. Here, the units of argon, $\sigma=3.405 \AA, \epsilon / k_{B}=125.2 \mathrm{~K}$, and $\tau=2.11 \mathrm{ps}$, were used. The values of the crystalline state $\lambda=1$ are comparable with experimental values for solid argon [30]. For $\lambda \leq 0.90$, the $T$ dependence becomes similar to the one observed in amorphous materials, with a power-law like increase $\kappa_{\text {real }} \sim T^{1.8}$ at low temperatures $(T<10[K])$, followed by a plateau above $T \sim 10[K]$. Note that $\kappa$ displays this "glassy" behaviour already in the disordered crystalline states with $0.90 \geq \lambda \geq \lambda^{*} \simeq 0.81$. This observation is consistent with experimental results reported by Cahill et al. 31]. These authors measured the thermal conductivity of disordered crystals, for example $(\mathrm{KBr})_{1-x}(\mathrm{KCN})_{x}$, by changing the composition $x . x=0$ corresponds to the crystalline state, and as $x$ increases to 0.5 , more disorder is introduced, so that $x$ plays a role similar to $\lambda$. As $x$ was increased, $\kappa$ was found to decrease steeply and to exhibit a glass-like $T$ dependence even in disordered crystals.

Conclusions and remarks. - In this work, we have used the flexibility offered by a numerical approach to study the correlations between elastic heterogeneity, vibrational states, and thermal conductivity in a model in which those quantities can be controlled continuously. We have been able to investigate in a unified framework the cases of the perfect crystal state, increasingly disordered crystals, and the isotropic amorphous solid state. We find that the heterogeneities of the bulk modulus $K^{m}$ and the 
highest shear modulus $G_{s}^{m}$ are well correlated with change in nature of the high frequency vibrational states. As $\lambda$ decreases from 1 to $0.9, K^{m}$ and $G_{s}^{m}$ become heterogeneous progressively, while the high $\omega$ modes loose their propagative character, leading to the reduction of the longitudinal branch in $g(\omega)$. The appearance of these heterogeneities is also correlated to a steep decrease of thermal conductivity $\kappa$, as the high frequency modes become non-propagating.

On the other hand, the lowest shear modulus $G_{p}^{m}$, which vanishes at the amorphisation point, was observed to be correlated with the low $\omega$ vibrational states. As $\lambda$ goes to $\lambda^{*}, G_{p}^{m}$ gets increasingly heterogeneous. Accompanying this heterogeneity, the excess of modes in $g(\omega) / \omega^{2}$ becomes pronounced, and at the same time, the low $\omega$ modes get localized. After the transition $\lambda^{*}$, the heterogeneity of $G_{p}^{m}$ converges to that of amorphous states immediately, and the excess peak in $g(\omega) / \omega^{2}$ also converges to the usual Boson peak.

We finalize this letter with a few remarks. The diverging behavior of $g(\omega) / \omega^{2}$ as $\lambda \rightarrow \lambda^{*}$ is very similar as that reported in jamming systems 32, where as the system goes to an unjamming point, $g(\omega)$ approaches a non-zero value, i.e., $g(\omega) / \omega^{2}$ diverges, in the limit of zero $\omega$. However, in our system, $g(\omega)$ always approaches a zero value even at $\lambda=0.81 \simeq \lambda^{*}$, where $g(\omega) \sim \omega^{1.5}$ is observed in our $\omega$ window. As one possibility, the difference may come from that only $G_{p}^{m}$ is heterogeneous around a vanishing value, $G_{p} \simeq 0$, in our system, whereas in jamming systems, both $G_{p}^{m}$ and $G_{s}^{m}$ vanish simultaneously.

A most striking result is that $\kappa$ is almost unchanged through the transition $\lambda^{*}$, indicating that $\kappa$, in a classical system, is not directly related to excess modes and low $\omega$ localizations. This decoupling may be partially a result of the classical nature of our simulations, where all vibrational modes are excited equally 33 . In real quantum systems, only low $\omega$ modes are excited according to the Bose-Einstein statistics, so that $\kappa$ is expected to be more influenced by the low $\omega$ excitations. In fact, calculations using a quantum approach based on the mode diffusivity 29 suggested a correlation between the Boson peak and the anomalous thermal conductivity 34$]$. This correlation is not apparent here, and from the classical calculation it appears that all the modes (including the low frequency ones) have already lost their heat transport capability before they become localized and the amorphisation threshold is reached.

As a final remark, we emphasize that the modulus distribution, the vibrational states, and the thermal conductivity are almost insensitive to disorder once the amorphisation threshold has been reached. In contrast, we observed important quantitative changes in the disordered crystalline states $1>\lambda>\lambda^{*}$. This suggests that introducing disorder into crystals may be a valuable strategy to control material properties, rather than attempting to control the amorphous state. In fact, synthetic nanostructuring in crystals is considered to be most promising way for controlling thermal conductivity 35].
Supplemental Material. - This is supplemental material which integrates the published paper.

Local elastic modulus.- The local elastic modulus $C_{i j k l}^{m}$ is defined as the first-derivative of the local stress $\sigma_{i j}^{m}$ with respect to the local strain $\epsilon_{k l}^{m}$ [17]. One possible implementation of the direct calculation of $C_{i j k l}^{m}$ is in terms of the equilibrium fluctuation formulae 17, 18. In Ref. 17], we referred to this method as the "Fully local approach", which proceeds as follows.

The simulation box is partitioned into $20^{3}$ cubic domains, identified by the index " $m$ " and of linear size $W=2 a=3.16$. Here, $a=1.58$ is the length of the unit cell for the FCC crystal. For each cube $m$, the local modulus tensor $C_{i j k l}^{m}$ is obtained as the sum of four terms: the Born term, $C_{B i j k l}^{m}$, the kinetic contribution, $C_{K i j k l}^{m}$, the pressure correction, $C_{C i j k l}^{m}$, and the fluctuation term, $-C_{F i j k l}^{m}$ :

$C_{i j k l}^{m}=C_{B i j k l}^{m}+C_{K i j k l}^{m}+C_{C i j k l}^{m}-C_{F i j k l}^{m}=C_{A i j k l}^{m}-C_{F i j k l}^{m}$

Here, $C_{A i j k l}^{m}=C_{B i j k l}^{m}+C_{K i j k l}^{m}+C_{C i j k l}^{m}$ corresponds to the response of a system which deforms affinely, while $-C_{F i j k l}^{m}$ accounts for the non-affinity of the deformation. The terms in Eq. (1) are evaluated via the equations:

$$
\begin{aligned}
& C_{B i j k l}^{m}= \frac{1}{W^{3}}\left\langle\sum_{a<b}\left(\frac{\partial^{2} \phi^{a b}}{\partial r^{a b^{2}}}-\frac{1}{r^{a b}} \frac{\partial \phi^{a b}}{\partial r^{a b}}\right)\right. \\
&\left.\times \frac{r_{i}^{a b} r_{j}^{a b} r_{k}^{a b} r_{l}^{a b}}{r^{a b^{2}}} \frac{q^{a b}}{r^{a b}}\right\rangle, \\
& C_{K i j k l}^{m}=2\left\langle\hat{\rho}^{m}\right\rangle T\left(\delta_{i k} \delta_{j l}+\delta_{i l} \delta_{j k}\right), \\
& C_{C i j k l}^{m}=-\frac{1}{2}\left(2\left\langle\sigma_{i j}^{m}\right\rangle \delta_{k l}-\left\langle\sigma_{i k}^{m}\right\rangle \delta_{j l}-\left\langle\sigma_{i l}^{m}\right\rangle \delta_{j k}\right. \\
&\left.\quad-\left\langle\sigma_{j k}^{m}\right\rangle \delta_{i l}-\left\langle\sigma_{j l}^{m}\right\rangle \delta_{i k}\right), \\
& C_{F i j k l}^{m}=\frac{V}{T}\left[\left\langle\sigma_{i j}^{m} \sigma_{k l}\right\rangle-\left\langle\sigma_{i j}^{m}\right\rangle\left\langle\sigma_{k l}\right\rangle\right] .
\end{aligned}
$$

Here, \langle\rangle represents the ensemble average, $\hat{\rho}^{m}$ is the local number density in the cube $m, r_{i}^{a b}$ denotes the vector joining particles $a$ and $b$, and $r^{a b}$ is their distance. The quantity $q^{a b}$ represents the length of the line segment $r_{i}^{a b}$ which is located inside the cube $m$. If the vector $r_{i}^{a b}$ is located outside cube $m$, it follows $q^{a b}=0$. The term $q^{a b} / r^{a b}$ therefore determines the contribution of each pairwise interaction to the Born term $C_{B i j k l}^{m}$. The local stress $\sigma_{i j}^{m}$ and the global stress $\sigma_{i j}$ are calculated as

$$
\begin{aligned}
\sigma_{i j}^{m} & =-\hat{\rho}^{m} T \delta_{i j}+\frac{1}{W^{3}} \sum_{a<b} \frac{\partial \phi^{a b}}{\partial r^{a b}} \frac{r_{i}^{a b} r_{j}^{a b}}{r^{a b}} \frac{q^{a b}}{r^{a b}}, \\
\sigma_{i j} & =\frac{1}{V} \sum_{m} W^{3} \sigma_{i j}^{m}=-\hat{\rho} T \delta_{i j}+\frac{1}{V} \sum_{a<b} \frac{\partial \phi^{a b}}{\partial r^{a b}} \frac{r_{i}^{a b} r_{j}^{a b}}{r^{a b}},
\end{aligned}
$$

where $\hat{\rho}=1.015$ is the global number density.

From the modulus tensor $C_{i j k l}^{m}$, we obtain the local bulk modulus $K^{m}$ and five local shear moduli $G_{1}^{m}, G_{2}^{m}, G_{3}^{m}$, 


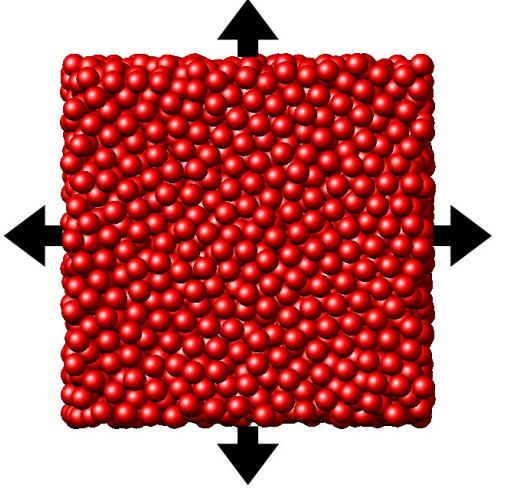

(a) Bulk deformation

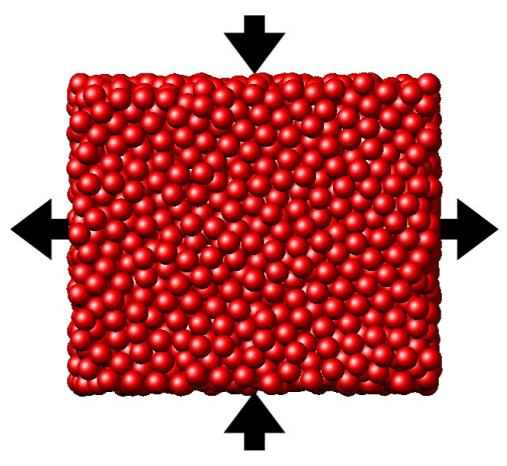

(b) Pure shear deformation

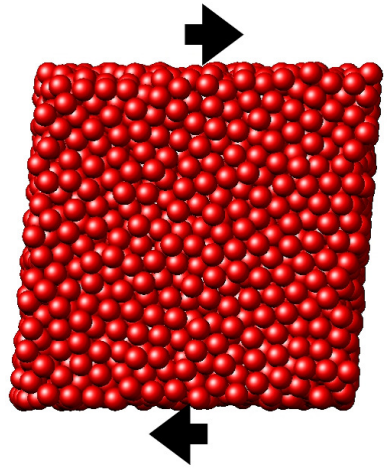

(c) Simple shear deformation

Fig. 7: Schematic illustration of (a) bulk deformation, (b) pure shear deformation, and (c) simple shear deformation.
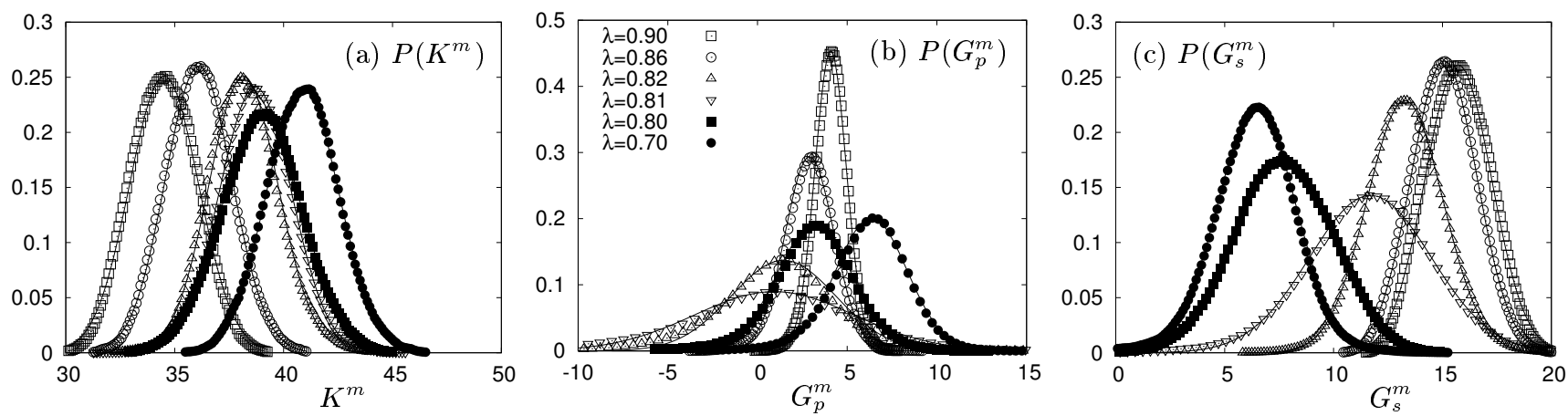

Fig. 8: Probability distribution of (a) bulk modulus $K^{m}$, (b) pure shear modulus $G_{p}^{m}$, and (c) simple shear modulus $G_{s}^{m}$. We also show the Gaussian distribution functions fitted to the calculated probability distributions (solid lines).

$G_{4}^{m}$, and $G_{5}^{m}$ from the following equations [17]:

$$
\begin{aligned}
3 K^{m}= & \left(C_{x x x x}^{m}+C_{y y y y}^{m}+C_{z z z z}^{m}+C_{x x y y}^{m}+C_{y y x x}^{m}\right. \\
& \left.+C_{x x z z}^{m}+C_{z z x x}^{m}+C_{y y z z}^{m}+C_{z z y y}^{m}\right) / 3, \\
2 G_{1}^{m}= & \left(C_{x x x x}^{m}+C_{y y y y}^{m}-C_{x x y y}^{m}-C_{y y x x}^{m}\right) / 2, \\
2 G_{2}^{m}= & \left(C_{x x x x}^{m}+C_{y y y y}^{m}+4 C_{z z z z}^{m}+C_{x x y y}^{m}+C_{y y x x}^{m}\right. \\
& \left.-2 C_{x x z z}^{m}-2 C_{z z x x}^{m}-2 C_{y y z z}^{m}-2 C_{z z y y}^{m}\right) / 6, \\
2 G_{3}^{m}= & C_{x y x y}^{m}, \\
2 G_{4}^{m}= & C_{x z x z}^{m}, \\
2 G_{5}^{m}= & C_{y z y z}^{m},
\end{aligned}
$$

Here, $G_{1}^{m}$ and $G_{2}^{m}$ correspond to "pure" shear deformations (plane and tri-axial strain deformations), and the remaining $G_{3}^{m}, G_{4}^{m}$, and $G_{5}^{m}$ are related to "simple" shear deformations. We give a schematic illustration for bulk deformation, pure shear deformation, and simple shear deformation in Fig. 77. In the Letter, we represent $G_{p}^{m}$ for $G_{1}^{m}$ and $G_{2}^{m}$, and $G_{s}^{m}$ for $G_{3}^{m}, G_{4}^{m}$, and $G_{5}^{m}$.

In Fig. 8, we show the probability distributions of local elastic moduli $K^{m}, G_{p}^{m}$, and $G_{s}^{m}$ at the indicated values of $\lambda$. The distributions are well described by Gaussian fitting functions (solid lines). From the distributions, we extract the average values, $K, G_{p}$, and $G_{s}$, and the standard de- viations, $\delta K^{m}, \delta G_{p}^{m}$, and $\delta G_{s}^{m}$, which are discussed in the Letter.

Visualization of vibrational modes.- The Letter also includes a detailed analysis of the nature of vibrational modes across the amorphisation transition. In particular, the degree of localization has been discussed in terms of participation ratios (see Fig.5). It is also instructive to directly visualize a few eigen-vectors. We plot in Fig. 9 the displacement fields corresponding to the indicated eigen-frequencies, for $\lambda=1.0,0.9,0.81$, and 0.8 . We have considered a system of size $L=10 a$ with $N=4,000$ particles. At each $\lambda$, we plot typical vibrational modes extracted from the low and high frequency parts of the spectrum, respectively. In the case of localized modes with a participation ratio $P R_{k}<0.1$, the largest $1 \%$ displacements are plotted as red arrows. In the perfect crystal case, $\lambda=1.0$, vibrational modes are extended and delocalized in the entire spectrum, as shown in Fig. 9(a) and (b). As $\lambda$ decreases, high frequency vibrational modes become increasingly localized (see Fig. 5), as it is evident for the case $\lambda=0.9$, in Fig. 9 (d). Below $\lambda=0.82$, localization also occurs at low frequencies (Fig. $9(\mathrm{f}-\mathrm{i})$ ). 


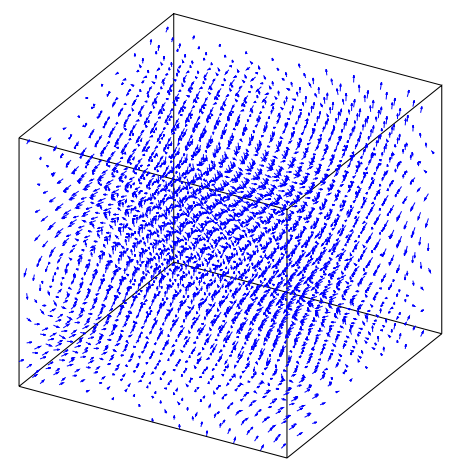

(a) $\lambda=1.0, \omega_{k}=1.27, P R_{k}=0.70$

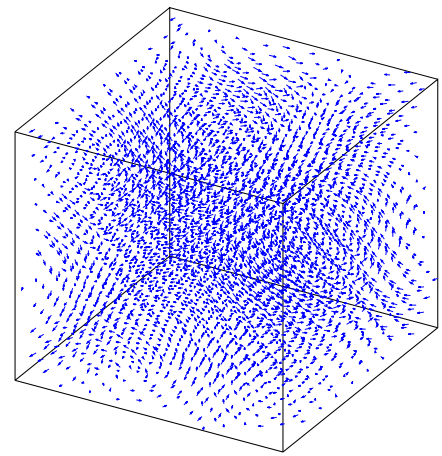

(c) $\lambda=0.9, \omega_{k}=1.09, P R_{k}=0.71$

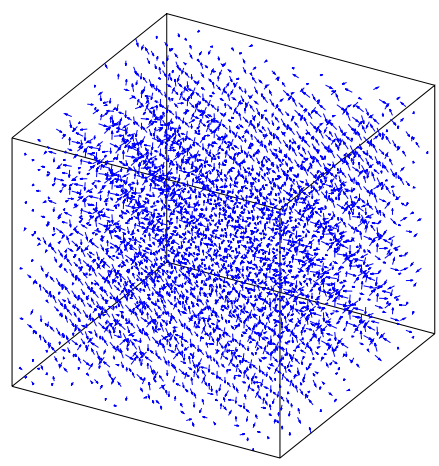

(b) $\lambda=1.0, \omega_{k}=15.9, P R_{k}=0.70$

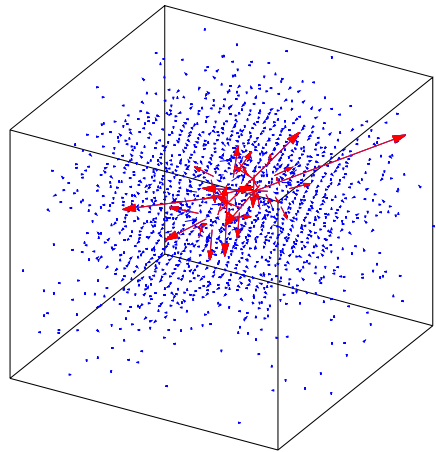

(d) $\lambda=0.9, \omega_{k}=18.8, P R_{k}=0.0019$

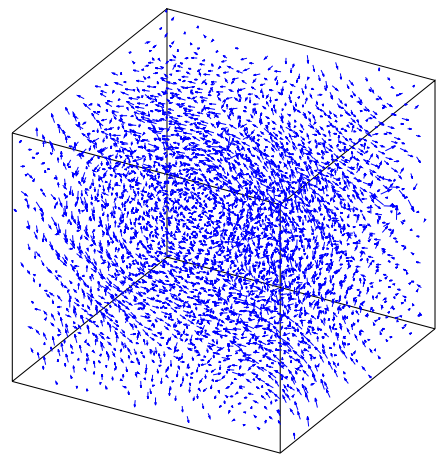

(e) $\lambda=0.81, \omega_{k}=0.43$,

$P R_{k}=0.60$

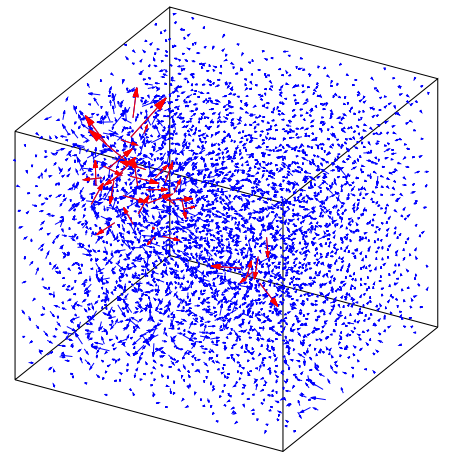

(h) $\lambda=0.8, \omega_{k}=0.58, P R_{k}=0.061$

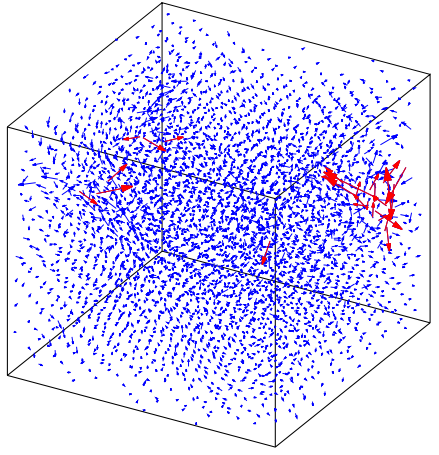

(f) $\lambda=0.81, \omega_{k}=0.82$,

$P R_{k}=0.066$

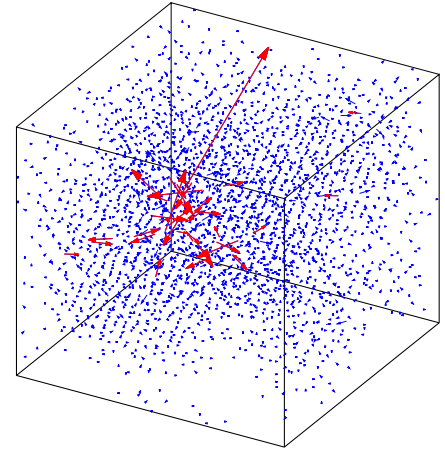

(g) $\lambda=0.81, \omega_{k}=21.9$, $P R_{k}=0.0015$

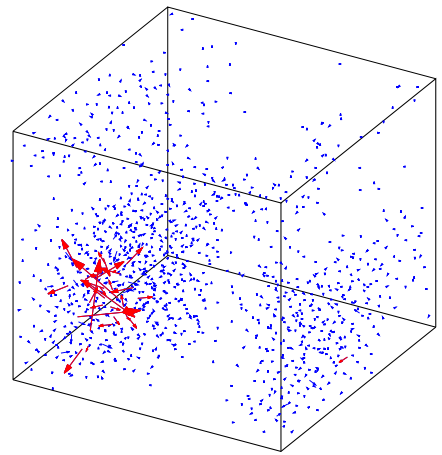

(i) $\lambda=0.8, \omega_{k}=21.9, P R_{k}=0.0017$

Fig. 9: Displacement field corresponding to a few eigen-vectors of the Hessian matrix: (a), (b) for $\lambda=1.0$, (c), (d) for $\lambda=0.9$, (e), (f), (g) for $\lambda=0.81$, and (h), (i) for $\lambda=0.8$. Arrows correspond to the displacements of particles We plotted the eigen-vectors of the particles and are multiplied by a factor of 10 , for clarity. We also indicate the eigen-frequency $\omega_{k}$ and the participation ratio $P R_{k}$ for each considered mode. In (d), (f), (g), (h), and (i), corresponding to localized modes with $P R_{k}<0.1$, the largest $1 \%$ displacements are plotted as red arrows. 

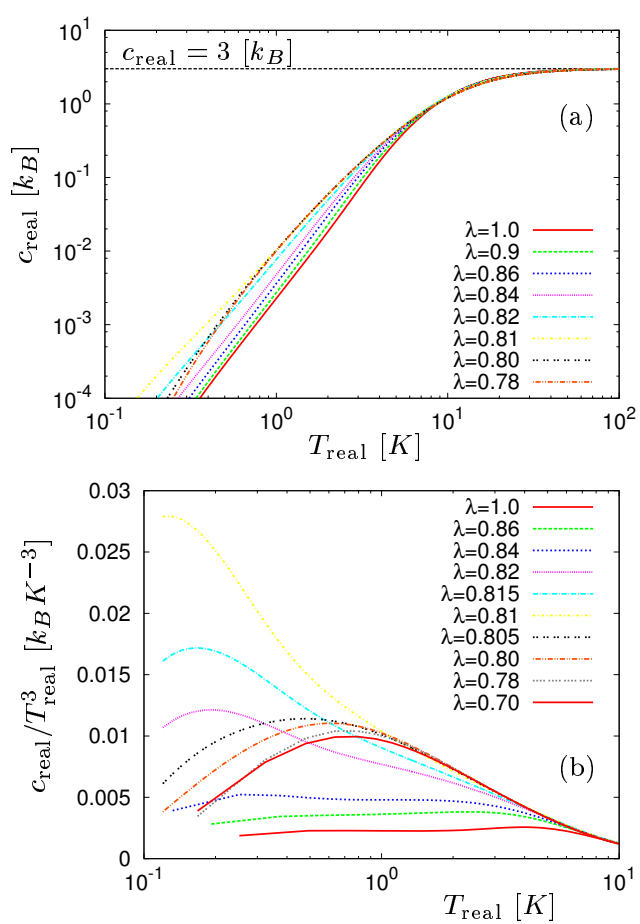

Fig. 10: The temperature $T_{\text {real }}$ dependence of (a) $c_{\text {real }}$ and (b) $c_{\text {real }} / T_{\text {real }}^{3}$.

Quantum corrections for thermal conductivity.Classical molecular dynamics simulations cannot obviously grasp the temperature dependence of heat capacity, which is a genuine quantum effect. However, it is possible to qualitatively incorporate quantum corrections to the study of thermal conductivity (see, among others, Ref. 27). In what follows all quantities are expressed in "Argon" units, $\sigma=3.405 \AA, \epsilon / k_{B}=125.2 \mathrm{~K}$, and $\tau=2.11 \mathrm{ps}$. The "classical" values of temperature, $T_{\mathrm{MD}}$ and thermal conductivity, $\kappa_{\mathrm{MD}}$, which are obtained from molecular dynamics simulation, can be mapped onto the "real" values of $T_{\text {real }}$ and $\kappa_{\text {real }}$. The value of $T_{\mathrm{MD}}$ is mapped onto $T_{\text {real }}$ by equating the energies of classical and quantum system:

$$
k_{B} T_{\mathrm{MD}}=\int \hbar \omega\left(\frac{1}{2}+\frac{1}{\exp \left(\hbar \omega / k_{B} T_{\text {real }}\right)-1}\right) g(\omega) d \omega .
$$

Here, $\hbar=h / 2 \pi, h$ is the Planck constant, and $g(\omega)$ is the vibrational density of state, which is shown in Fig. 3(a). The first term of right-hand side in Eq. (5) is the "zero point energy" of the quantum system. In the present study, we have not taken into account the zero point energy.

Mapping of $\kappa_{\mathrm{MD}}$ onto $\kappa_{\text {real }}$ is expressed by

$$
\kappa_{\text {real }}=\frac{C_{\text {real }}}{C_{\mathrm{MD}}} \kappa_{\mathrm{MD}}
$$

where $C_{\mathrm{MD}}$ and $C_{\text {real }}$ are the heat capacities of classical and quantum systems, respectively. The value $C_{\text {real }}$ is calculated as

$$
\begin{aligned}
c_{\text {real }} & =\frac{C_{\text {real }}}{N-1} \\
& =3 k_{B} \int \frac{\left(\hbar \omega / k_{B} T_{\text {real }}\right)^{2} \exp \left(\hbar \omega / k_{B} T_{\text {real }}\right)}{\left(\exp \left(\hbar \omega / k_{B} T_{\text {real }}\right)-1\right)^{2}} g(\omega) d \omega,
\end{aligned}
$$

where $c_{\text {real }}$ is the heat capacity per particle. In Fig. 10(a), we show $c_{\text {real }}$ as a function of $T_{\text {real }}$. As $T_{\text {real }}$ increases, $c_{\text {real }}$ tends to the classical value $3 k_{B}$. For the perfect crystal case, $\lambda=1.0, c_{\text {real }} \sim T_{\text {real }}^{3}$ in the low temperature regime, e.g., the Debye-model prediction holds. However, as $\lambda$ decreases, the temperature dependence of $c_{\text {real }}$ deviates from the Debye model. We plot $c_{\text {real }} / T_{\text {real }}^{3}$ in Fig. 10 (b). We clearly see that the peak value in $c_{\text {real }} / T_{\text {real }}^{3}$ is pronounced at the transition point $\lambda=\lambda^{*} \simeq 0.81$. This effect originates from the excess of low frequency modes in $g(\omega) / \omega^{2}$, as shown in Fig. 3(b).

$$
* * *
$$

We acknowledge useful discussions with Dr. O. N. Bedoya-Martinez. This work was supported by the Nanosciences Foundation of Grenoble. JLB is supported by the Institut Universitaire de France.

\section{REFERENCES}

[1] S.R. Elliott, Physics of amorphous materials (Wiley, New York) 1984.

[2] W.A. Phillips, Amorphous Solids: Low Temperature Properties (Springer, Berlin) 1981.

[3] M.I. Klinger, Phys. Rep., 94 (1983) 183.

[4] W. Schirmacher et al., Phys. Rev. Lett., 81 (1998) 136.

[5] T. S. Grigera et al., Nature, 422 (2003) 289.

[6] M. Wyart, Annales de Physiques,3020051

[7] E. Duval and A. Mermet, Phys. Rev. B, 5819988159

[8] W. Schirmacher, Europhys. Lett., 73 (2006) 892.

[9] W. Schirmacher et al., Phys. Rev. Lett., 98 (2007) 025501.

[10] K. Yoshimoto et al., Phys. Rev. Lett., 93 (2004) 175501.

[11] M. Tsamados et al., Phys. Rev. E, 80 (2009) 026112.

[12] A. Makke et al., Macromol. Theory Simul., 20 (2011) 826.

[13] F. Leonforte et al., Phys. Rev. B, 72 (2005) 224206.

[14] A. Marruzzo et al., Nature Scientific Reports, 3 (2013) 1407.

[15] L. Bocquet et al., J. Phys.: Condens. Matter, 4 (1992) 2375 .

[16] B. Bernu et al., Phys. Rev. A, 36 (1987) 4891.

[17] H. Mizuno et al., Phys. Rev. E, 87 (2013) 042306.

[18] J. F. Lutsko, J. Appl. Phys., 64 (1988) 1152.

[19] http://arxiv.org/abs/1308.5135

[20] A. Tanguy et al., Phys. Rev. B, 66 (2002) 174205.

[21] Cz. Jasiukiewicz and V. Karpus, Solid State Commun., 128 (2003) 167.

[22] G. Monaco And S. Mossa, Proc. Natl. Acad. Sci. USA, 106 (2009) 16907.

[23] V. Mazzacurati et al., Europhys. Lett., 34 (1996) 681. 
[24] H. R. Schober And G. Ruocco, Philos. Mag., 84 (2004) 1361.

[25] H. Shintani And H. TAnaka, Nature Mater., 7 (2008) 870.

[26] P. Tan et al., Phys. Rev. Lett., 108 (2012) 095501.

[27] A. J. H. McGaughey and M. Kaviany, Advances in Heat Transfer, edited by G. A. Greene, Y. I. Cho, J. P. Hartnett, And A. Bar-Cohen (Elsevier, New York) 2006, Vol. 39, p. 169-255.

[28] C. Masciovecchio et al., Phys. Rev. Lett., 97 (2006) 035501.

[29] P. B. Allen and J.L. Feldman, Phys. Rev. B, 48 (1993) 12581 .

[30] D. K. Christensen and G.L. Pollack, Phys. Rev. B, 12 (1975) 3380.

[31] D. G. Cahill et al., Phys. Rev. B, 46 (1992) 6131.

[32] L. E. Silbert et al., Phus. Rev. Lett., 95 (2005) 098301.

[33] The quantum correction we applied is a global one, which does not take into account the mode-dependent occupation discussed in J. E. Turney et al., Phys. Rev. B, 79 (2009) 224305 .

[34] N. Xu et al., Phys. Rev. Lett., 102 (2009) 038001.

[35] J. MA et al., Nature Nanotech., 8 (2013) 445. 